The University of San Francisco

USF Scholarship: a digital repository @ Gleeson Library |

Geschke Center

Physics and Astronomy

College of Arts and Sciences

12-15-1999

\title{
Electrical Resistivity of a Thin Metallic Film
}

Horacio E. Camblong

University of San Francisco, camblong@usfca.edu

Follow this and additional works at: http://repository.usfca.edu/phys

Part of the Physics Commons

\section{Recommended Citation}

Camblong, Horacio E., "Electrical Resistivity of a Thin Metallic Film" (1999). Physics and Astronomy. Paper 9.

http://repository.usfca.edu/phys/9

This Article is brought to you for free and open access by the College of Arts and Sciences at USF Scholarship: a digital repository @ Gleeson Library | Geschke Center. It has been accepted for inclusion in Physics and Astronomy by an authorized administrator of USF Scholarship: a digital repository @ Gleeson Library | Geschke Center. For more information, please contact repository@usfca.edu. 


\title{
Electrical resistivity of a thin metallic film
}

\author{
Horacio E. Camblong \\ Department of Physics, University of San Francisco, San Francisco, California 94117 \\ Peter M. Levy \\ Department of Physics, New York University, New York, New York 10003
}

(Received 19 July 1999)

\begin{abstract}
The electrical resistivity of a pure sample of a thin metallic film is found to depend on the boundary conditions. This conclusion is supported by a free-electron model calculation and confirmed by an ab initio relativistic Korringa-Kohn-Rostoker computation. The low-temperature resistivity is found to be zero for a free-standing film (reflecting boundary conditions) but nonzero when the film is sandwiched between two semi-infinite samples of the same material (outgoing boundary conditions). In the latter case, this resistivity scales inversely with the number of monolayers and is due to the background diffusive scattering by a finite lattice. [S0163-1829(99)00147-2]
\end{abstract}

\section{INTRODUCTION}

It is well known that the low-temperature electrical resistivity of an infinite pure metal is essentially zero because the Bloch waves associated with the underlying periodic structure undergo no diffusive scattering in the absence of impurities and imperfections. Then, one could ask what the corresponding result would be for a thin metallic film. This question, which has not been addressed in the literature, is the central problem tackled in this paper. Our conclusion is that the electrical resistivity of a thin film depends upon how the experimental situation is set up, namely, upon the boundary conditions; in particular, with an appropriate choice of boundary conditions, the resistivity is not zero.

The problem of the electrical resistivity of a pure sample of a thin metallic film is of great current interest. It can be viewed as a fundamental question for which an exploratory analytic calculation may provide deeper insight as well as a limiting test of complex $a b$ initio methods. In fact, this question can be posed in the context of the first-principle fully relativistic layered version of the Korringa-Kohn-Rostoker (KKR) method, which has been recently developed, ${ }^{1}$ to perform $a b$ initio computations of magnetotransport in magnetic multilayers in the coherent-potential approximation. In effect, this method can be easily applied to the computation of the electrical resistivity of a thin film consisting of a finite number $M$ of monolayers of a metal, by properly specifying the conditions enforced at the boundaries of the system. The first result was obtained for a thin film of copper sandwiched between two semi-infinite metallic blocks of the same material; the resistivity was computed for a film consisting of between $M=6$ and 45 monolayers and for current in the plane of the layers (CIP) and was found to decrease approximately as $116 / M \mu \Omega \mathrm{cm}$, for $M>20 \mathrm{ML}$ - a result that clearly extrapolates to zero only as $M$ approaches infinity. This nonzero value is somewhat unusual and led us to ask that the computation be done under different boundary conditions. For a free-standing slab, namely, when the copper film is surrounded by vacuum on both sides, it was found that the resistivity is essentially zero. ${ }^{2}$ In conclusion, when one performs these two computations with unequal boundary conditions, one gets unequal resistivities. In other words, the resistivity depends on the boundary conditions.

The results described above raise a number of questions that we address in this paper. Our goal is to explore electrical conduction in a pure sample of a thin metallic film and resolve the following fundamental issues. (i) Is the resistivity indeed dependent on the boundary conditions? (ii) How can one understand the finite resistivity of a thin, yet otherwise perfect, film? (iii) How can one measure the finite resistivity of a thin film?

As we will see in Sec. II, a proper understanding of boundary conditions is a prerequisite for a thorough discussion of these questions. This analysis will be followed in Sec. III by the application of the Kubo formula to the case with outgoing boundary conditions, in Sec. IV by the mathematical characterization of the finite periodicity of the lattice, in Sec. V by the implications of the film finiteness on transport properties, and in Sec. VI by concluding remarks.

\section{RESISTIVITY AND BOUNDARY CONDITIONS}

Electrical conduction in a thin metallic film can be modeled by representing the film in terms of a finite number $M$ of monolayers or perfect atomic planes arranged periodically in the direction perpendicular to the boundaries of the film. Moreover, a pure sample is characterized by the absence of impurities or imperfections; in addition, at low temperatures, other resistivity sources are rendered ineffective.

At first sight, one might just apply the standard folklore, namely, that quantum-mechanical Bloch waves in a periodic structure propagate without electrical resistance. In effect, electrical resistance arises from the loss of linear momentum information due to diffusive scattering, i.e., scattering that randomizes the electron's momentum, so that the outgoing electron has no knowledge of the direction of its incoming momentum. ${ }^{3}$ In fact, in a pure metallic sample, electrons undergo Bragg scattering, which being highly directional, is ineffective as a momentum-randomizing mechanism (as we will discuss in greater detail in Sec. V). This would seem to imply that the resistivity of a perfect film should be identi- 
cally zero; however, this line of reasoning is simplistic: the standard folklore applies only to an infinite periodic sample, for which the electrical resistivity is indeed zero, under the conditions described above. Instead, when one analyzes a finite film, the question "What is the resistivity of a thin metallic film?" is immediately replaced by "Is the film really periodic?', Then, from a mathematical viewpoint, the film fails to be strictly periodic because of the boundaries; by abuse of language, one could describe the film as exhibiting "finite periodicity." It turns out that the finiteness of the film implies the existence of background diffusive scattering in addition to ordinary Bragg scattering (see Sec. V), and it is this diffusive scattering that becomes the source of a finitesize resistivity if the boundary conditions are appropriately selected.

The simplest boundary condition is provided by the ideal free-standing slab when a film with perfect boundaries is inserted in a vacuum. Then, the electrons undergo specular reflections at the boundaries (with infinitely high potentials representing the onset of a vacuum) and effectively "probe" a truly periodic potential. This amounts to repeating the film periodically; periodicity is restored by the boundary conditions, and the standard folklore applies: the resistivity $\rho$ is indeed zero. In fact, the absence of electrical resistivity can be traced back again to the electrons keeping their memory of linear momentum. In the old Fuchs-Sondheimer transport model, ${ }^{4}$ this reflecting boundary condition, which amounts to the absence of diffuse scattering at the boundaries $(100 \%$ specular reflection), is parametrized by $p=1$, where $p$ is the coefficient characterizing the specularity of scattering off the surfaces of the film. The ensuing resistivity $\rho=0$ has been confirmed by the relativistic layered KKR method. ${ }^{2}$

Does this mean that the resistivity is always zero? Of course not; the same relativistic layered KKR method showed that finite-size effects are not negligible when the film is sandwiched between two semi-infinite samples of the same material. The novelty lies here in the use of different boundary conditions. In effect, if the boundary conditions dictate that the electrons cannot keep their memory of momentum, then the background scattering of the finite lattice becomes the source of a nonzero electrical resistivity.

An extreme form of this loss of momentum information is achieved when the momentum of electrons entering the finite sample is totally uncorrelated with that of electrons leaving the sample. One can conceptualize this extreme loss of momentum information in three equivalent ways: in terms of reservoirs, in terms of boundary scattering, and in terms of boundary conditions for the electron propagators (Green's functions). First, momentum loss is effectively implemented by having reservoirs that absorb the electrons upon leaving the sample, when the electrons probe a reservoir, they get out of synch with respect to their "proper" behavior in the sample. Second, from the scattering viewpoint, this information loss can be modeled by perfectly diffuse scattering at the boundaries of the film; this condition is precisely equivalent to the choice $p=0$ in the Fuchs-Sondheimer model ${ }^{4}(0 \%$ specular reflection, a condition that totally erases momentum memory). The third viewpoint is needed when applying the Kubo formula in terms of electron propagators; in our original theory of transport in metallic superlattices, ${ }^{5}$ we described the required condition associated with this momen- tum loss as outgoing boundary conditions.

Transport theory with outgoing boundary conditions is based on the following ideas. Transport is described via retarded Green's functions that represent the propagation of electrons in the environment provided by the sample, with the condition that the electrons leave the film irreversibly at the boundaries. This implies the use of Green's functions corresponding to an infinite medium (bulk) — at this level the calculation does not acknowledge the finiteness of the film whose conductivity is calculated. Then, as the propagators themselves do not satisfy boundary conditions that keep track of the momentum of electrons scattered within the film, the calculation yields a nonzero resistivity (see Sec. V). In other words, all finite conductors have self-energy terms in their propagators that describe their contact with leads or reservoirs. The resistance, which is proportional to the imaginary part of the self-energy, reflects the fact that an electron in a finite conductor will eventually leak out into the leads attached to it. ${ }^{6}$

The discussion above has dealt successfully with the first two questions posed in Sec. I: the resistivity is indeed dependent on the boundary conditions, and we have understood conceptually how the finite resistivity of a perfect thin film arises under outgoing boundary conditions. However, the issue of how to measure the finite resistivity of a thin film has not yet been clarified. From the experimental viewpoint, the finite electrical resistivity of a perfect thin film still remains puzzling, even when the concept of finite periodicity is introduced. In effect, if we isolate the film from a bulk sample of copper and maintain it in contact with the "remainder" of the system (two perfectly conducting semi-infinite copper blocks), then the current is shunted by these contacts. In other words, it would seem that it is not possible to measure the resistivity of the film in this straightforward way. In fact, one concludes that, for a measurement of electrical resistivity, the boundary contacts should not be of the same material.

So how does one observe the calculated resistance? As mentioned in the previous paragraph, the shunting of the current by the contacts prohibits one from measuring the resistance of a portion of a metal. However, if the current probe is sufficiently narrow to contact only the finite layer, and appropriate boundary conditions are enforced, then the current will be limited only to the finite layer. Then, one can either measure the resistance of a free-standing film or have it supported on an insulating substrate. If the boundaries are ideal so that they have no appreciable roughness, i.e., for $p$ $=1$, the boundaries simulate reflecting or free-standing boundary conditions and lead to zero resistivity. Alternatively, if the boundaries are sufficiently roughened, they simulate current flow only in the finite layer subject to the $p=0$ boundary condition-this amounts to outgoing boundary conditions. In fact, the roughened interface could even separate the finite layer from a semi-infinite sample of the same material on either side, a situation that is modeled by the corresponding relativistic KKR computation. ${ }^{2}$ It is in this way that we understand the paradox of using a film with a rough surface to measure the resistance calculated for a perfectly flat film; it is the boundary condition for transport on the surface of the film that happens to be the same in both cases. 
A parenthetical remark is in order. The statement that $p$ $=0$ corresponds to outgoing boundary conditions should not signify that the resistivity coming from the "bulk" of the sample is directly related to the scattering at the boundaries; the latter are there merely to simulate the boundary conditions that enable the "bulk" scattering to produce resistance. In other words, the surface should have a roughness profile sufficient to guarantee the boundary condition $p=0$; however, further increasing the amplitude of the roughness, while increasing the resistivity due to the surface scattering, does not increase the resistivity coming from the bulk scattering. Therefore, for the case at hand, the actual scattering does not come from randomly situated impurities but from the potential of the positively charged background ions that form a finite, but otherwise perfect, lattice. A perfect periodic lattice has no resistivity, and it is easy to overlook the fact that electrons are scattered by it, i.e., they undergo Bragg scattering. As we will show in Sec. V, a finite but otherwise perfect lattice scatters electrons for all momenta, with two main contributions: constructive interference or Bragg scattering as well as background diffusive scattering. It is the latter that leads to electrical resistivity and vanishes in the infinite-thickness limit; unlike the case of impurity scattering, its resistivity is inversely proportional to the thickness of the film.

In the remainder of this paper, we will show the details of the calculation of the electrical resistivity of a thin film using the Kubo formula within the free-electron model. Specifically, we will show that outgoing boundary conditions do indeed imply the existence of a finite electrical resistivity. Remarkably, the free-electron result agrees in form and reasonably well in magnitude with the one calculated $a b$ initio by Blaas et al. ${ }^{1,2}$ for a perfect slab of copper embedded in copper.

\section{KUBO FORMALISM FOR THE CONDUCTIVITY OF A THIN METALLIC SLAB}

As discussed in Sec. II, a perfectly diffuse boundary amounts to outgoing boundary conditions, which are implemented with the corresponding infinite-medium retarded Green's functions. As the scattering ultimately leading to resistivity is due to a potential $V(\mathbf{r})$ with "finite periodicity," we resolve the Hamiltonian in the form

$$
H=H_{0}+V(\mathbf{r}),
$$

where the unperturbed Hamiltonian $H_{0}$ corresponds to free electrons characterized by the eigenfunctions $\varphi_{\mathbf{k}}$ $=\Omega^{-1 / 2} e^{i \mathbf{k} \cdot \mathbf{r}}$; in all quantum-mechanical computations, we will use particle-in-a-box normalization with finite volume $\Omega$. However, given a thin film of cross-sectional area $A$, thickness $L$, and volume $\Omega=A L$, the in-plane dimensions (defining the area $A$ ) will be effectively regarded as infinite, whereas the finiteness of the perpendicular or "longitudinal" dimension $L$ will become the source of finite-size effects; this longitudinal direction will be chosen to correspond to the $z$ axis. Accordingly, whenever appropriate, a generic vector $\mathbf{V}$ will be resolved into its in-plane component $\mathbf{V}_{\|}$and its longitudinal component $\mathbf{V}_{\perp}=\hat{\mathbf{z}} V_{z}$; with this notation, the energy of the free-particle or unperturbed state of momentum $\hbar \mathbf{k}$ is

$$
\epsilon_{\mathbf{k}}=\epsilon_{\mathbf{k} \| k_{z}}=\epsilon_{\mathbf{k}_{\|}}+\epsilon_{k_{z}}
$$

where $\epsilon_{\mathbf{k}_{\|}}=\hbar^{2} k_{\|}^{2} / 2 m$ and $\epsilon_{k_{z}}=\hbar^{2} k_{z}^{2} / 2 m$, with $m$ being the effective electron mass. Our calculation of electrical conductivity will be performed by applying perturbation theory within the framework of the Kubo formula. ${ }^{7,8}$ The required potential matrix elements are given by

$$
V_{\mathbf{k k}^{\prime}}=\frac{1}{\Omega} \int d^{3} r e^{-i\left(\mathbf{k}-\mathbf{k}^{\prime}\right) \cdot \mathbf{r}} V(\mathbf{r})=\frac{1}{\Omega} \widetilde{V}\left(\mathbf{k}-\mathbf{k}^{\prime}\right),
$$

where $\widetilde{V}(\mathbf{k})$ is the Fourier transform of the potential. Then, to second order in perturbation theory, the diagonal momentum-space elements of the $t$ matrix are

$$
t_{\mathbf{k}}(\epsilon)=V_{\mathbf{k k}}+\sum_{\mathbf{k}^{\prime}} V_{\mathbf{k k}^{\prime}} G_{\mathbf{k}^{\prime}}^{0}(\epsilon) V_{\mathbf{k}^{\prime} \mathbf{k}},
$$

where $G^{0}(\epsilon)$ is the free-particle propagator. In order to evaluate the Kubo formula for the electrical conductivity, the negative imaginary part of the $t$ matrix, $\Delta=-\operatorname{Im}(t)$ is needed. Then, from the condition $V_{\mathbf{k k}^{\prime}}^{*}=V_{\mathbf{k}^{\prime} \mathbf{k}}$, it follows that

$$
\begin{aligned}
\Delta\left(\mathbf{k}_{\|}, k_{z} ; \boldsymbol{\epsilon}\right) & =\Delta(\mathbf{k} ; \boldsymbol{\epsilon}) \\
& =-\operatorname{Im}\left[t_{\mathbf{k}}(\boldsymbol{\epsilon})\right] \\
& =\pi \sum_{\mathbf{k}_{\|}^{\prime}, k_{z}^{\prime}} \mathcal{J}\left(\mathbf{k}-\mathbf{k}^{\prime}\right) \delta\left(\epsilon-\epsilon_{\mathbf{k}_{\|}^{\prime}}-\epsilon_{k_{z}^{\prime}}\right),
\end{aligned}
$$

in which the effect of the potential on the conductivity is now summarized by the function

$$
\mathcal{J}(\mathbf{q})=\frac{|\widetilde{V}(\mathbf{q})|^{2}}{\Omega^{2}},
$$

with $\mathbf{q}=\mathbf{k}-\mathbf{k}^{\prime}$ and where the density of states $\delta\left(\epsilon-\epsilon_{\mathbf{k}_{\|}^{\prime}}\right.$ $\left.-\epsilon_{k_{z}^{\prime}}\right)$ has been directly extracted from $-\operatorname{Im}\left[G_{\mathbf{k}_{\|}^{\prime} k_{z}^{\prime}}^{0}(\epsilon)\right] / \pi$.

The Kubo formula ${ }^{7}$ gives the zero-temperature in-plane (CIP) dc conductivity in terms of the in-plane current-current correlation function by means of the formal double-limit expression $^{8}$

$$
\sigma=\lim _{\beta \rightarrow \infty} \lim _{\omega \rightarrow 0} \frac{1}{2 \omega \Omega}\left[\int_{0}^{\beta} d \tau e^{i \omega \tau}\left\langle T_{\tau} \mathbf{j} \|(\tau) \cdot \mathbf{j}_{\|}(0)\right\rangle\right]_{i \omega \rightarrow \omega+i 0^{+}},
$$

which in the independent-electron approximation reduces straightforwardly to the familiar form

$$
\sigma=\frac{e^{2}}{\Omega} \sum_{\mathbf{k}_{\|}, k_{z}, s} \frac{1}{2}\left[\frac{\partial \epsilon_{\mathbf{k}_{\|} k_{z}}}{\partial\left(\hbar k_{\|}\right)}\right]^{2} \frac{\hbar}{2 \Delta\left(\mathbf{k}_{\|}, k_{z} ; \epsilon_{F}\right)} \delta\left(\epsilon_{F}-\epsilon_{\mathbf{k}_{\|}}-\epsilon_{k_{z}}\right),
$$

where $s$ stands for the electron-spin index. Equation (8) can be conveniently rewritten by evaluating the group velocity as

$$
\left[\frac{\partial \epsilon_{\mathbf{k}_{\|}}}{\partial\left(\hbar \mathbf{k}_{\|}\right)}\right]^{2}=\frac{2 \epsilon_{\mathbf{k}_{\|}}}{m},
$$

and applying the in-plane continuum limit 


$$
\frac{1}{A} \sum_{\mathbf{k}_{\|}, s} \rightarrow 2 \int \frac{d^{2} \mathbf{k}_{\|}}{(2 \pi)^{2}}=\int d \epsilon_{\mathbf{k}_{\|}} g_{\|}\left(\epsilon_{\mathbf{k}_{\|}}\right)
$$

with the only restriction that the integrand be a spinindependent function of the momentum variables $\left|\mathbf{k}_{\|}\right|$and $k_{z}$ (but not of the corresponding angular in-plane variable). In Eq. (10), $g_{\|}\left(\epsilon_{\mathbf{k}_{\|}}\right)$is the in-plane two-dimensional (2D) density of states per spin degree of freedom and per unit area, which is given by

$$
g_{\|}(\epsilon)=\frac{1}{4 \pi} \frac{2 m}{\hbar^{2}},
$$

provided that the in-plane dimensions be effectively infinite. Notice that this density of states $g_{\|}(\epsilon)$ is actually a constant $g_{\|}$, which from now on will be factored out of the corresponding integrals. Then, Eqs. (8)-(11) imply that the conductivity is given by the expression

$$
\sigma=\frac{e^{2}}{h} \frac{1}{L} \sum_{k_{z}} \frac{\epsilon_{F}-\epsilon_{k_{z}}}{\Delta\left(k\left(\epsilon_{F}-\epsilon_{k_{z}}\right), k_{z} ; \epsilon_{F}\right)},
$$

where $k(\epsilon)$ is the positive root of the equation $\epsilon_{k}=\epsilon$, i.e., $k(\epsilon)=\sqrt{2 m \epsilon} / \hbar$; in particular,

$$
k\left(\epsilon_{F}-\epsilon_{k_{z}}\right)=\sqrt{k_{F}^{2}-k_{z}^{2}} .
$$

In Eq. (12), it is assumed that $\Delta\left(\mathbf{k}_{\|}, k_{z} ; \boldsymbol{\epsilon}_{F}\right)$ is a function of $\left|\mathbf{k}_{\|}\right|$and $k_{z}$ alone; thus, to simplify the notation, from now on this quantity will be represented as $\Delta\left(\left|\mathbf{k}_{\|}\right|, k_{z} ; \epsilon_{F}\right)$.

Equation (12) will be the starting point for our conductivity calculation in Sec. V, where we will straightforwardly apply its counterpart in the longitudinal continuum limit,

$$
\begin{aligned}
\frac{1}{L} \sum_{k_{z}} f\left(k_{z}\right) \rightarrow & \int_{-\infty}^{\infty} \frac{d k_{z}}{2 \pi} f\left(k_{z}\right)=\frac{1}{2} \int_{0}^{\infty} d \epsilon_{k_{z}} g_{\perp}\left(\epsilon_{k_{z}}\right) \\
& \times\left[f\left(k\left(\epsilon_{k_{z}}\right)\right)+f\left(-k\left(\epsilon_{k_{z}}\right)\right)\right],
\end{aligned}
$$

where

$$
g_{\perp}(\epsilon)=\frac{1}{2 \pi}\left(\frac{2 m}{\hbar^{2}}\right)^{1 / 2} \epsilon^{-1 / 2}
$$

is the longitudinal density of states per spin degree of freedom and per unit length; notice that, explicitly, $k\left(\epsilon_{k_{z}}\right)$ $=\left|k_{z}\right|$. Under this approximation, Eq. (12) turns into

$$
\begin{aligned}
\sigma= & \frac{e^{2}}{2 h} \int_{0}^{\epsilon_{F}} d \epsilon_{k_{z}}\left(\epsilon_{F}-\epsilon_{k_{z}}\right) g_{\perp}\left(\epsilon_{k_{z}}\right)\left[\frac{1}{\Delta\left(k\left(\epsilon_{F}-\epsilon_{k_{z}}\right), k\left(\epsilon_{k_{z}}\right) ; \epsilon_{F}\right)}\right. \\
& \left.+\frac{1}{\Delta\left(k\left(\epsilon_{F}-\epsilon_{k_{z}}\right),-k\left(\epsilon_{k_{z}}\right) ; \epsilon_{F}\right)}\right] .
\end{aligned}
$$

\section{FINITE PERIODICITY}

A thin film can be regarded as built out of primitive cells assembled into an effectively infinite arrangement in two directions (for which we will use symbols 1 and 2) and a finite layering in a third direction (for which we will use the symbol 3). As we will consider a generic "finite Bravais lattice," the three directions need not be perpendicular to each other. In other words, if the number of primitive cells stacked in direction $j$ is $N_{j}$, then $N_{1}, N_{2} \gg N_{3}$, and, in practice, we will regard $N_{1}$ and $N_{2}$ as effectively infinite but $N_{3}$ as a finite number; notice that $N=N_{1} N_{2} N_{3}$ is the total number of primitive cells in the film. The finite periodicity in the third direction can be described by counting the number $M=N_{3}$ $+1 \approx N_{3}$ of stacked infinite lattice planes or monolayers; notice that, for the sake of simplicity, we will assume $M \gg 1$. For instance, if the film has thickness $L$ and consists of exactly $M$ monolayers from one boundary to the other, then $L$ $=N_{3} d \approx M d$, with $d$ being the distance between consecutive monolayers.

The basic periodicity of the crystal structure within its boundaries can be described by means of a finite generalization of the concept of Bravais lattice. As usual, given the primitive translation vectors $\mathbf{a}_{j}$, with $j=1,2,3$, it follows that the set of all translation vectors $\mathbf{R}$ is of the form $\mathbf{R}=n_{1} \mathbf{a}_{1}$ $+n_{2} \mathbf{a}_{2}+n_{3} \mathbf{a}_{3}$, where the integers $n_{j}$ are limited to the values $0 \leqslant n_{j} \leqslant N_{j}-1$ for a finite lattice. Then, for any local function of the position, such as the lattice potential, the property

$$
V(\mathbf{r}+\mathbf{R})=V(\mathbf{r})
$$

remains valid within the boundaries of the film. In particular, for Fourier transforms, finite periodicity guarantees the identity

$$
\int_{T_{\mathbf{R}} \mathcal{C}} d^{3} r e^{-i \mathbf{q} \cdot \mathbf{r}} V(\mathbf{r})=e^{-i \mathbf{q} \cdot \mathbf{R}} \int_{\mathcal{C}} d^{3} r e^{-i \mathbf{q} \cdot \mathbf{r}} V(\mathbf{r}),
$$

where an arbitrary primitive cell $\mathcal{C}$ can be related to any other primitive cell via a translation $T_{\mathbf{R}}$ by a Bravais lattice vector R. This property leads to the characteristic Bragg peaks with respect to electronic conduction, as shown below. In effect, the Fourier transform of the potential becomes

$$
\begin{aligned}
\widetilde{V}(\mathbf{q})= & \int_{\mathcal{V}} d^{3} r e^{-i \mathbf{q} \cdot \mathbf{r}} V(\mathbf{r})=\sum_{n_{1}=0}^{N_{1}-1} \sum_{n_{2}=0}^{N_{2}-1} \sum_{n_{3}=0}^{N_{3}-1} \\
& \times e^{-i \mathbf{q} \cdot\left(n_{1} \mathbf{a}_{1}+n_{2} \mathbf{a}_{2}+n_{3} \mathbf{a}_{3}\right)} \int_{\mathcal{C}} d^{3} r e^{-i \mathbf{q} \cdot \mathbf{r}} V(\mathbf{r}),
\end{aligned}
$$

where $\mathcal{V}$ is the entire sample and $\mathcal{C}$ is a reference primitive cell. Then, summing the geometric progressions involved in Eq. (19) and replacing in Eq. (6), one finds

$$
\mathcal{J}(\mathbf{q})=\left[\prod_{j=1}^{3} \mathcal{F}_{N_{j}}\left(\mathbf{q} \cdot \mathbf{a}_{j}\right)\right] \mathcal{J}^{(0)}(\mathbf{q})
$$

where 


$$
\mathcal{J}^{(0)}(\mathbf{q})=\left|\int_{\mathcal{C}} \frac{d^{3} r}{\mathbf{V}} e^{-i \mathbf{q} \cdot \mathbf{r}} V(\mathbf{r})\right|^{2}
$$

is the corresponding quantity for just one primitive cell, $v$ $=\mathbf{a}_{1} \cdot\left(\mathbf{a}_{2} \times \mathbf{a}_{3}\right)$ is the volume of a primitive cell, and

$$
\mathcal{F}_{N_{j}}\left(\xi_{j}\right)=\frac{1}{N_{j}^{2}}\left[\frac{\sin \left(N_{j} \xi_{j} / 2\right)}{\sin \left(\xi_{j} / 2\right)}\right]^{2}
$$

stands for the interference factor associated with $N_{j}$ identical primitive cells aligned in the direction of the primitive translation vector $\mathbf{a}_{j}$, with

$$
\xi_{j}=\mathbf{q} \cdot \mathbf{a}_{j} .
$$

The interference factors are the origin of the Bragg scattering peaks, which are represented by $\delta$ functions and amount to the selection of the conditions $\xi_{j}=\mathbf{q} \cdot \mathbf{a}_{j}=2 \pi n_{j}$ (with $n_{j}$ integer numbers), as can be seen from the vanishing of the denominator in Eq. (22). These $\delta$ functions can be explicitly displayed by means of the expansion in a series of simple fractions

$$
\frac{1}{\sin ^{2}\left(\xi_{j} / 2\right)}=\sum_{n_{j}=-\infty}^{\infty} \frac{1}{\left(\xi_{j} / 2-n_{j} \pi\right)^{2}}
$$

and from the familiar asymptotic result

$$
\frac{1}{N_{j}}\left[\frac{\sin \left(N_{j} \xi_{j} / 2\right)}{\left(\xi_{j} / 2\right)}\right]^{2} \sim \pi \delta\left(\xi_{j} / 2\right)
$$

for $N_{j} \rightarrow \infty$; then, the interference factors become

$$
\begin{aligned}
\mathcal{F}_{N_{j}}\left(\xi_{j}\right) & =\frac{1}{N_{j}^{2}} \sum_{n_{j}=-\infty}^{\infty}\left[\frac{\sin \left(N_{j} \xi_{j} / 2\right)}{\left(\xi_{j} / 2-n_{j} \pi\right)}\right]^{2} \\
& \sim \frac{2 \pi}{N_{j}} \sum_{n_{j}=-\infty}^{\infty} \delta\left(\xi_{j}-2 \pi n_{j}\right),
\end{aligned}
$$

where the second expression is to be understood as the asymptotic form for $N_{j}$ "sufficiently large." The continuum limit implicit in Eq. (26) can be reversed by replacing the Dirac delta function by a Kronecker delta and recalling Eq. (23), whence

$$
\mathcal{F}_{N_{j}}\left(\xi_{j}\right) \sim \sum_{n_{j}=-\infty}^{\infty} \delta_{\mathbf{q} \cdot \mathbf{a}_{j}, 2 \pi n_{j}} .
$$

In particular, the combination of the three structure factors in Eq. (20) amounts to

$$
\prod_{j=1}^{3} \mathcal{F}_{N_{j}}\left(\xi_{j}\right) \sim \sum_{n_{1}, n_{2}, n_{3}=-\infty}^{\infty} \delta_{\mathbf{q} \cdot \mathbf{a}_{1}, 2 \pi n_{1}} \delta_{\mathbf{q} \cdot \mathbf{a}_{2}, 2 \pi n_{2}} \delta_{\mathbf{q} \cdot \mathbf{a}_{3}, 2 \pi n_{3}} .
$$

Equation (28) can be reinterpreted by expanding $\mathbf{q}$ in terms of primitive reciprocal vectors, i.e., $\mathbf{q}=\nu_{1} \mathbf{b}_{1}+\nu_{2} \mathbf{b}_{2}+\nu_{3} \mathbf{b}_{3}$, with $\mathbf{a}_{j} \cdot \mathbf{b}_{h}=2 \pi \delta_{j h}$, whence $\nu_{j}=\mathbf{q} \cdot \mathbf{a}_{j} / 2 \pi$. Then, Eq. (28) states that $\nu_{j}=n_{j}$ is an integer, so that $\mathbf{q}$ is indeed a reciprocal-lattice vector $\mathbf{G}_{n_{1} n_{2} n_{3}}$, i.e.,

$$
\prod_{j=1}^{3} \mathcal{F}_{N_{j}}\left(\xi_{j}\right) \sim \sum_{n_{1}, n_{2}, n_{3}=-\infty}^{\infty} \delta_{\mathbf{q}, \mathbf{G}_{n_{1} n_{2} n_{3}}} .
$$

However, in a finite lattice, it is legitimate to apply the limit of Eq. (29) only with respect to the directions defined by the primitive vectors $\mathbf{a}_{1}$ and $\mathbf{a}_{2}$. Then, the component of $\mathbf{q}$ on the plane spanned by the primitive reciprocal vectors $\mathbf{b}_{1}$ and $\mathbf{b}_{2}$ is

$$
\mathbf{q}_{\text {in }}=\mathbf{q}-\frac{\left(\mathbf{q} \cdot \mathbf{a}_{3}\right)}{2 \pi} \mathbf{b}_{3}=\mathbf{G}_{n_{1} n_{2} 0},
$$

which is a $2 \mathrm{D}$ reciprocal vector,

$$
\mathbf{g}_{n_{1} n_{2}}=\mathbf{G}_{n_{1} n_{2} 0}=n_{1} \mathbf{b}_{1}+n_{2} \mathbf{b}_{2},
$$

so that

$$
\prod_{j=1}^{2} \mathcal{F}_{N_{j}}\left(\xi_{j}\right) \sim \sum_{n_{1}, n_{2}=-\infty}^{\infty} \delta_{\mathbf{q}_{\mathrm{in}}, \mathbf{g}_{n_{1} n_{2}}} .
$$

Next we will assume that, for $\mathbf{q}=\mathbf{k}-\mathbf{k}^{\prime}$, with $\mathbf{k}$ and $\mathbf{k}^{\prime}$ on the Fermi surface, the only allowed 2D reciprocal-lattice vector $\mathbf{g}_{n_{1} n_{2}}$ is the zero vector. To see that this assumption is reasonable, let us consider, for example, a thin film of copper, for which one can choose the set of primitive vectors $\mathbf{a}_{1}=(a / 2)(\hat{\mathbf{x}}-\hat{\mathbf{y}}), \mathbf{a}_{2}=(a / 2)(\hat{\mathbf{x}}+\hat{\mathbf{y}}), \mathbf{a}_{3}=(a / 2)(\hat{\mathbf{x}}+\hat{\mathbf{z}}), \quad$ and primitive reciprocal vectors $\mathbf{b}_{1}=(2 \pi / a)(\hat{\mathbf{x}}-\hat{\mathbf{y}}-\hat{\mathbf{z}}), \mathbf{b}_{2}$ $=(2 \pi / a)(\hat{\mathbf{x}}+\hat{\mathbf{y}}-\hat{\mathbf{z}}), \mathbf{b}_{3}=(4 \pi / a) \hat{\mathbf{z}}$, with $a \approx 3.61 \AA$ and $k_{F}$ $\approx 1.36 \AA^{-1}$. Then, for $\mathbf{q}=\mathbf{k}-\mathbf{k}^{\prime}$, with $\mathbf{k}$ and $\mathbf{k}^{\prime}$ on the Fermi surface, it follows that

$$
|\mathbf{q}| \leqslant 2 k_{F}<\left|\mathbf{b}_{1}\right|,\left|\mathbf{b}_{2}\right|,
$$

implying that the only acceptable choice is $n_{1}=n_{2}=0$, namely, $\mathbf{g}_{n_{1} n_{2}}=\mathbf{0}$. Under these conditions, $\mathbf{q}_{\text {in }}=\mathbf{0}$ and also $\mathbf{q}_{\|}=\mathbf{0}$, because $\mathbf{q} \cdot \mathbf{a}_{1}=0$ and $\mathbf{q} \cdot \mathbf{a}_{2}=0$ simultaneously; thus, $\mathbf{q}=\mathbf{q}_{\perp}$. Then, the argument of the interference factor $\mathcal{F}_{N_{3}}\left(\xi_{3}\right)$ is

$$
\xi_{3}=\mathbf{q}_{\perp} \cdot \mathbf{a}_{3}=q_{z} d,
$$

with $d$ being the distance between consecutive monolayers. As a consequence, Eq. (20) becomes

$$
\mathcal{J}(\mathbf{q})=\mathcal{J}^{(\perp)}\left(q_{z}\right) \delta_{\mathbf{q}_{\|}, \mathbf{0}},
$$

where

$$
\mathcal{J}^{(\perp)}\left(q_{z}\right)=\mathcal{F}_{N_{3}}\left(q_{z} d\right) \mathcal{J}^{(0)}\left(q_{z} \hat{\mathbf{z}}\right),
$$

with

$$
\mathcal{J}^{(0)}\left(q_{z} \hat{\mathbf{z}}\right)=\left|\int_{\mathcal{C}} \frac{d^{3} r}{\mathbf{V}} V(\mathbf{r}) e^{-i q_{z} z}\right|^{2}
$$

[cf. Eq. (21)]. Equations (35)-(37) give just a Bragg scattering contribution and zero resistivity as $N_{3} \rightarrow \infty$, but fall short of that singular behavior for $N_{3}$ finite. Based on the preceding analysis, the Bragg scattering contributions, $\mathcal{J}_{\text {Bragg }}(\mathbf{q})$ and $\mathcal{J}_{\text {Bragg }}^{(\perp)}\left(q_{z}\right)$, are defined to be the asymptotic forms of the functions $\mathcal{J}(\mathbf{q})$ and $\mathcal{J}^{(\perp)}\left(q_{z}\right)$ as $N_{3} \rightarrow \infty$; thus, they are related by Eq. (35), with 


$$
\mathcal{J}_{\text {Bragg }}^{(\perp)}\left(q_{z}\right)=\frac{2 \pi}{N_{3}} \sum_{n_{3}=-\infty}^{\infty} \delta\left(q_{z} d-2 \pi n_{3}\right) \mathcal{J}^{(0)}\left(\frac{2 \pi n_{3}}{d} \hat{\mathbf{z}}\right)
$$

in particular,

$$
\begin{aligned}
& {\left[\mathcal{J}_{\text {Bragg }}\left(\mathbf{q}_{\|}=\mathbf{0}, q_{z}\right)\right]_{\left|q_{z}\right|<2 \pi / d}=\left[\mathcal{J}_{\text {Bragg }}^{(\perp)}\left(q_{z}\right)\right]_{\left|q_{z}\right|<2 \pi / d}} \\
& \quad=\frac{2 \pi}{N_{3}} \delta\left(q_{z} d\right) \mathcal{J}^{(0)}(\mathbf{0}),
\end{aligned}
$$

an expression that will be important in the derivation of the electrical resistivity in the next section.

\section{FINITE ELECTRICAL RESISTIVITY}

Equation (12) will give a nonzero electrical resistivity only when the $t$ matrix develops a nonzero imaginary part, i.e., $\Delta_{\mathbf{k}_{\|} k_{z}}(\epsilon) \neq 0$. This imaginary part may arise in the process of replacing discrete sums by energy integrals [according to the rule defined by Eq. (14)], if the self-energy acquires an analytic structure characterized by a branch cut that is effectively generated by the merging of the discrete poles of the discrete self-energy. However, the scattering by a periodic lattice generates constructive interference in discrete directions (represented by $\delta$ functions); this Bragg scattering fails to produce an imaginary self-energy.

Due to the directional nature of Bragg scattering, the term $\mathcal{J}_{\text {Bragg }}^{(\perp)}\left(k_{z}-k_{z}^{\prime}\right)$ given in Eq. (38) does not contribute to the sum of Eq. (5), namely,

$$
-\sum_{\mathbf{k}_{\|}^{\prime}, k_{z}^{\prime}} \mathcal{J}_{\text {Bragg }}\left(\mathbf{k}-\mathbf{k}^{\prime}\right) \operatorname{Im}\left[G_{\mathbf{k}_{\|}^{\prime} k_{z}^{\prime}}^{0}(\epsilon)\right]=0 .
$$

Then, we are led to a resolution of the function $\mathcal{J}(\mathbf{q})$ of Eq. (6) into two parts,

$$
\mathcal{J}(\mathbf{q})=\mathcal{J}_{\text {Bragg }}(\mathbf{q})+\mathcal{J}_{\text {diff }}(\mathbf{q})=\left[\mathcal{J}_{\text {Bragg }}^{(\perp)}\left(q_{z}\right)+\mathcal{J}_{\text {diff }}^{(\perp)}\left(q_{z}\right)\right] \delta_{\mathbf{q}_{\|}, \mathbf{0}},
$$

corresponding to Bragg scattering, given by Eq. (38), and the remainder, which we identify as background diffusive scattering. This procedure, which is based on the analysis of Sec. IV, amounts to isolating the diffusive part of the scattering $\mathcal{J}_{\text {diff }}(\mathbf{q})=\mathcal{J}(\mathbf{q})-\mathcal{J}_{\text {Bragg }}(\mathbf{q})$, which leads to a finite resistivity via the term

$$
\begin{aligned}
\Delta\left(\mathbf{k}_{\|}, k_{z} ; \epsilon\right) & =\pi \sum_{\mathbf{k}_{\|}^{\prime}, k_{z}^{\prime}} \mathcal{J}_{\text {diff }}\left(\mathbf{k}-\mathbf{k}^{\prime}\right) \delta\left(\epsilon-\epsilon_{\mathbf{k}_{\|}^{\prime}}-\epsilon_{k_{z}^{\prime}}\right) \\
& =\pi \sum_{k_{z}^{\prime}} \mathcal{J}_{\text {diff }}^{(\perp)}\left(k_{z}-k_{z}^{\prime}\right) \delta\left(\epsilon-\epsilon_{\mathbf{k}_{\|}}-\epsilon_{k_{z}^{\prime}}\right) .
\end{aligned}
$$

Equation (41) can be further simplified by either applying the longitudinal continuum approximation, Eq. (14) with respect to $z^{\prime}$, or explicitly rewriting the $\delta$ function as

$$
\begin{aligned}
\delta\left(\epsilon-\epsilon_{\mathbf{k}}\right)= & 2 \pi g^{(\perp)}\left(\epsilon-\epsilon_{\mathbf{k}_{\|}}\right) \\
& \times \frac{1}{2}\left[\delta\left(k_{z}-k\left(\epsilon-\epsilon_{\mathbf{k}_{\|}}\right)\right)+\delta\left(k_{z}+k\left(\epsilon-\epsilon_{\mathbf{k}_{\|}}\right)\right)\right]
\end{aligned}
$$

$$
=\frac{L}{2} g^{(\perp)}\left(\boldsymbol{\epsilon}-\epsilon_{\mathbf{k}_{\|}}\right)\left[\delta_{k_{z}, k\left(\epsilon-\epsilon_{\mathbf{k}_{\|}}\right)}+\delta_{k_{z},-k\left(\epsilon-\epsilon_{\mathbf{k}_{\|}}\right.}\right] .
$$

Then,

$$
\begin{aligned}
\Delta\left(\mathbf{k}_{\|}, k_{z} ; \epsilon\right)= & \frac{\pi L}{2} g_{\perp}\left(\epsilon-\epsilon_{\mathbf{k}_{\|}}\right)\left[\mathcal{J}_{\text {diff }}^{\perp}\left(k_{z}-k\left(\epsilon-\epsilon_{\mathbf{k}_{\|}}\right)\right)\right. \\
& \left.+\mathcal{J}_{\text {diff }}^{\perp}\left(k_{z}+k\left(\epsilon-\epsilon_{\mathbf{k}_{\|}}\right)\right)\right] .
\end{aligned}
$$

Finally, for the calculation of the conductivity, Eqs. (12) and (16) dictate that Eq. (43) be evaluated for electrons on the Fermi surface, for which the conditions $\left|k_{z}\right|=k\left(\epsilon_{F}-\epsilon_{\mathbf{k}_{\mid}}\right)$and $k_{\|}=k\left(\epsilon_{F}-\epsilon_{k_{z}}\right)$ apply; then,

$$
\Delta\left(k\left(\epsilon_{F}-\epsilon_{k_{z}}\right), k_{z} ; \epsilon_{F}\right)=\frac{\pi L}{2} g_{\perp}\left(\epsilon_{k_{z}}\right)\left[\mathcal{J}_{\text {diff }}^{(\perp)}(0)+\mathcal{J}_{\text {diff }}^{(\perp)}\left(2 k_{z}\right)\right] .
$$

Substitution of Eq. (44) into Eq. (16) leads to

$$
\sigma=\frac{e^{2}}{h} \frac{2}{\pi L} \int_{0}^{\epsilon_{F}} d \epsilon_{k_{z}} \frac{\epsilon_{F}-\epsilon_{k_{z}}}{\mathcal{J}_{\text {diff }}^{(\perp)}(0)+\mathcal{J}_{\text {diff }}^{(\perp)}\left(2 k_{z}\right)},
$$

where the properties $\mathcal{J}_{\text {diff }}^{(\perp)}\left( \pm 2 k_{z}\right)=\left[\mathcal{J}_{\text {diff }}^{(\perp)}\left(2 k_{z}\right)\right]^{*}$ have been applied. Finally, the term $\mathcal{J}_{\text {diff }}^{(\perp)}\left(2 k_{z}\right)$ can be approximated with its value $\mathcal{J}_{\text {diff }}^{(\perp)}(0)=\mathcal{J}_{\text {diff }}(\mathbf{0})$, because it is partially suppressed by the numerator as $k_{z}$ approaches $k_{F}$ (in fact, the exponential in the corresponding Fourier integral does not complete one entire cycle even as $z$ approaches $d$ and $k_{z}$ approaches $k_{F}$ and is approximated as taking the value one); then,

$$
\sigma \approx \frac{e^{2}}{h} \frac{\epsilon_{F}^{2}}{2 \pi L \mathcal{J}_{\text {diff }}(\mathbf{0})}
$$

In order to evaluate the final conductivity expression of Eq. (46), the value of

$$
\mathcal{J}_{\text {diff }}(\mathbf{0})=\lim _{q_{z} \rightarrow 0}\left[\mathcal{J}\left(\mathbf{q}_{\|}=\mathbf{0}, q_{z}\right)-\mathcal{J}_{\text {Bragg }}\left(\mathbf{q}_{\|}=\mathbf{0}, q_{z}\right)\right]
$$

is required. This can be obtained from the interference factor, Eq. (22), which can be approximated for $N_{3}$ large and $\xi_{3}$ small via the expansion

$$
\begin{aligned}
\mathcal{F}_{N_{3}}\left(\xi_{3}\right) & =\frac{1}{N_{3}^{2}} \frac{\sin ^{2}\left(N_{3} \xi_{3} / 2\right)}{\left(\xi_{3} / 2\right)^{2}}\left[1+\frac{2}{3 !}\left(\frac{\xi_{3}}{2}\right)^{2}\right]+O\left(\xi_{3}^{2}\right) \\
& \sim \frac{2 \pi}{N_{3}} \delta\left(\xi_{3}\right)+\frac{1}{3 N_{3}^{2}} \sin ^{2}\left(\frac{N_{3} \xi_{3}}{2}\right)+O\left(\xi_{3}^{2}\right),
\end{aligned}
$$

where the denominator has been expanded in power series of $\xi_{3} / 2$, and Eq. (25) has been applied to provide the asymptotic 
form of $\mathcal{F}_{N_{3}}\left(\xi_{3}\right)$. The derivation above focuses directly on the $\xi_{3} \rightarrow 0$ limit and does not emphasize the resolution of the spectrum into infinitely many Bragg peaks, each of which has a long tail that contributes to the background diffusive scattering. Instead, a more illuminating approach would be to display all the Bragg peaks explicitly from the start by considering the limit $\xi_{3} \rightarrow 0$ of the simple fraction expansion of Eq. (24), namely,

$$
\lim _{\xi_{3} \rightarrow 0}\left[\frac{1}{\sin ^{2}\left(\xi_{3} / 2\right)^{2}}-\frac{1}{\left(\xi_{3} / 2\right)^{2}}\right]=\frac{2}{\pi^{2}} \sum_{n=1}^{\infty} \frac{1}{n^{2}}=\frac{2}{\pi^{2}} \zeta(2)=\frac{1}{3},
$$

a result that is in agreement with Eqs. (47) and (48). In the formulas above, in the limit $M \rightarrow \infty$, the factor $\sin ^{2}\left(M \xi_{3} / 2\right)$ oscillates fast about its average value $1 / 2$, which would therefore be effectively achieved in all physical measurements; thus, one concludes that Eq. (48) is simplified to

$$
\mathcal{F}_{N_{3}}\left(\xi_{3}\right)=\frac{2 \pi}{N_{3}} \delta\left(\xi_{3}\right)+\frac{1}{6 N_{3}^{2}}+O\left(\xi_{3}^{2}\right)
$$

Notice the characteristic appearance of the Bernoulli number $B_{2}=1 / 6$, associated with either the expansion of Eq. (48) or with the value $\zeta(2)=B_{2} \pi^{2}$ of the Riemann $\zeta$ function in Eq. (49). Then, for $\xi_{3}=q_{z} d$ small, Eqs. (35), (36), (39), and (50) imply that

$$
\begin{aligned}
\mathcal{J}\left(\mathbf{q}_{\|}=\mathbf{0}, q_{z}\right)= & \mathcal{F}_{N_{3}}\left(q_{z} d\right) \mathcal{J}^{(0)}(\mathbf{0}) \\
= & {\left[\mathcal{J}_{\text {Bragg }}\left(\mathbf{q}_{\|}=\mathbf{0}, q_{z}\right)\right]_{\left|q_{z}\right|<2 \pi / d} } \\
& +\frac{1}{6 N_{3}^{2}} \mathcal{J}^{(0)}(\mathbf{0})+O\left(q_{z}^{2}\right) ;
\end{aligned}
$$

therefore, from Eqs. (37) and (47), and from $N_{3} \approx M$, the diffusive part of $\mathcal{J}(\mathbf{0})$ for $M$ monolayers is

$$
\mathcal{J}_{\text {diff }}(\mathbf{0})=\frac{1}{6 M^{2}} \mathcal{J}^{(0)}(\mathbf{0})=\frac{1}{6 M^{2}}\langle V\rangle^{2}
$$

with

$$
\langle V\rangle=\int_{\mathcal{C}} \frac{d^{3} r}{\mathrm{~V}} V(\mathbf{r})
$$

being the average potential.

Finally, replacing Eqs. (52) and (53) into Eq. (46), and recalling that $L \approx M d$, we get a remarkably simple expression for the resistivity,

$$
\rho \approx \frac{\pi}{3} \frac{h}{e^{2}} \frac{\Gamma^{2} d}{M}
$$

where

$$
\Gamma=\frac{1}{\epsilon_{F}} \int_{\mathcal{C}} \frac{d^{3} r}{\mathrm{~V}} V(\mathbf{r})=\left\langle\frac{V(\mathbf{r})}{\epsilon_{F}}\right\rangle,
$$

which is the average potential relative to the Fermi energy, is a dimensionless parameter characterizing the relative strength of the periodic potential.
Equation (54) can be evaluated numerically by introducing a natural atomic resistivity

$$
\rho_{0}=\frac{h}{e^{2}}(1 \AA) \approx 258 \mu \Omega \mathrm{cm},
$$

whence

$$
\rho \approx \frac{270 \Gamma^{2} d[\AA]}{M} \mu \Omega \mathrm{cm} .
$$

Equation (57) summarizes one of the main results of this paper. It displays a characteristic inverse proportionality with respect to the number $M$ of monolayers. In addition, notice that:

(i) The period $d$ is not a free parameter, as it is uniquely determined from the crystal structure.

(ii) As the atomic length scale $d[\AA]$ is always of the order of unity, the largest variations in the resistivity scale of Eq. (57) will come from the dimensionless parameter $\Gamma$.

(iii) $\Gamma$ is indeed the only free parameter within the framework of approximations used in this model.

(iv) The value of $\Gamma$ can be independently estimated from calculations of cohesive energy.

For example, for copper, reasonable estimates are provided by the following values of the relevant parameters: ${ }^{9}$ $d \approx 1.8 \AA$ and $\Gamma \approx 2 / 7$; then, the predicted CIP resistivity is approximately $40 / M \mu \Omega \mathrm{cm}$, a value reasonably close to the $116 / M \mu \Omega \mathrm{cm}$ found by the ab initio method. ${ }^{2}$

\section{CONCLUSIONS}

It is generally recognized that a perfect infinite periodic structure leads to no electrical resistance because of the condition of constructive interference or Bragg scattering. In this paper, we have shown that, at sufficiently low temperatures, for sufficiently clean samples, and for outgoing boundary conditions, finiteness of a metallic film will prevent the potential from being perfectly periodic, will produce an effective background diffusive scattering, and will cause a sizedependent resistivity inversely proportional to the number of monolayers. Our free-electron estimate is in close agreement with similar results from $a b$ initio calculations. It would be interesting to see if this resistivity can be measured experimentally by properly simulating outgoing (perfectly diffusive) boundary conditions.

As a coda, it is worth mentioning that the Boltzmann equation approach with the conventional relaxation time approximation fails for transport through a finite, yet otherwise perfect, film. The usual ansatz for the nonequilibrium distribution function ${ }^{10} f(\mathbf{k})=f_{0}(k)+\mathbf{k} \cdot \mathbf{E} C(k)$ is not applicable, because the deviation from equilibrium depends on the orientation of the electric field relative to the crystal axes of the film as well as on the angle between $\mathbf{k}$ and $\mathbf{E}$.

\section{ACKNOWLEDGMENTS}

This work was supported in part (P.M.L.) by the Office of Naval Research together with the Defense Advanced Research Projects Agency (Grant No. N00014-96-1-1207), the National Science Foundation (Grant No. INT-9602192), and NATO (Grant No. CRG 960340). 
${ }^{1}$ C. Blaas, P. Weinberger, L. Szunyogh, P. M. Levy, C. B. Sommers, and I. Mertig, Philos. Mag. B 78, 549 (1998); P. Weinberger, P. M. Levy, J. Banhart, L. Szunyogh, and B. Ujfalussy, J. Phys.: Condens. Matter 8, 7677 (1996).

${ }^{2}$ C. Blaas, P. Weinberger, L. Szunyogh, P. M. Levy, and C. B. Sommers, Phys. Rev. B 60, 492 (1999). The resistivities quoted in this publication should all be increased by a factor of four, P. Weinberger (private communication). Therefore the quoted resistivity for copper embedded in copper of $29 / M \mu \Omega \mathrm{cm}$ should read $116 / M \mu \Omega \mathrm{cm}$.

${ }^{3}$ S. Doniach and E. H. Sondheimer, Green's Functions for Solid State Physicists (Benjamin, Reading, MA, 1974).
${ }^{4}$ E. H. Sondheimer, Adv. Phys. 1, 1 (1952).

${ }^{5}$ H. E. Camblong, Phys. Rev. B 51, 1855 (1995).

${ }^{6}$ S. Datta, Electronic Transport in Mesoscopic Systems (Cambridge University Press, Cambridge, England, 1995).

${ }^{7}$ R. Kubo, J. Phys. Soc. Jpn. 12, 570 (1957).

${ }^{8}$ G. D. Mahan, Many-Particle Physics, 2nd ed. (Plenum, New York, 1990).

${ }^{9}$ F. Seitz, The Modern Theory of Solids (McGraw-Hill, New York, 1940), pp. 369-370; the approximate values given for $\epsilon_{F}$ and $\langle V\rangle$ are, respectively, 7 and $2 \mathrm{eV}$.

${ }^{10}$ G. D. Mahan, Many-Particle Physics (Ref. 8), pp. 602-606. 